\title{
Two Identification Methods for a Nonlinear Membership Function
}

\author{
Yuejiang Ji $(\mathbb{D}$ and Lixin Lv (D) \\ Wuxi Vocational College of Science and Technology, Wuxi 214122, China \\ Correspondence should be addressed to Yuejiang Ji; jyj1981917@126.com \\ Received 6 February 2021; Revised 28 March 2021; Accepted 31 March 2021; Published 3 May 2021 \\ Academic Editor: Quanmin Zhu \\ Copyright (c) 2021 Yuejiang Ji and Lixin Lv. This is an open access article distributed under the Creative Commons Attribution \\ License, which permits unrestricted use, distribution, and reproduction in any medium, provided the original work is \\ properly cited. \\ This paper proposes two parameter identification methods for a nonlinear membership function. An equation converted method \\ is introduced to turn the nonlinear function into a concise model. Then a stochastic gradient algorithm and a gradient-based \\ iterative algorithm are provided to estimate the unknown parameters of the nonlinear function. The numerical example shows \\ that the proposed algorithms are effective.
}

\section{Introduction}

Parameter estimation has many applications in system modelling and signal processing [1-5]. Recently, there exist many parameter estimation approaches, such as recursive least squares (RLS) methods [6-8], stochastic gradient (SG) methods [9-11], and iterative identification methods [12-14]. For example, Chen et al. proposed an iterative method for Hammerstein systems with saturation and dead-zone nonlinearities [15]. Li et al. presented a gradient iterative estimation algorithm and a Newton iterative estimation algorithm for a nonlinear function [16].

In the past years, the approximation capability of fuzzy systems has received much attention especially for control purposes [17-19]. The approximation theory of fuzzy systems is often applied to approximate the unknown functions of control systems. It is shown that the identification of membership functions plays an essential role to ensure the system capability. There are many different types of membership functions in the approximation theory of fuzzy systems, such as the Gaussian membership functions [20-22] and other membership functions $[23,24]$.

Since the membership functions usually have complex nonlinear structures, the traditional least squares may be infeasible for the identification of the functions because their derivative functions sometimes do not have analytical solutions [25-27]. Thanks to the gradient descent (GD) algorithm, which avoids solving an analytical function, thus it can be extended to complex nonlinear membership functions. The GD algorithm generates a sequence estimate using an iterative function, where the iterative function consists of two parts: one is the negative direction and the other is the step size [28, 29]. With a correct direction and an optimal step size, the GD algorithm can ensure the results converge to the true values.

In this paper, we propose two methods to estimate the unknown parameters of a nonlinear membership function; these two methods are both based on the GD method. First, a gradient iterative algorithm is proposed, which can estimate the parameters based on all the collected data, and thus, it has heavy computational efforts. In order to reduce the computational efforts, we turn the model of the nonlinear function into a regression model and then use two identification algorithms to estimate the unknown parameters.

The rest of the paper is organized as follows. Section 2 introduces the nonlinear function and the gradient-based iterative algorithm. Section 3 develops the model transformation-based stochastic gradient method and iterative method. Section 4 provides an illustrative example. Finally, concluding remarks are given in Section 5. 


\section{The Nonlinear Membership Function and the Gradient-Based Iterative Algorithm}

Let us introduce some notations first. The symbol I stands for an identity matrix of the appropriate sizes; the norm of a matrix $\mathbf{X}$ is defined as $\|\mathbf{X}\|:=\sqrt{\left(\left(\mathbf{X}^{T} \mathbf{X}\right)\right)}$; the superscript $T$ denotes the matrix transpose.

Consider a nonlinear membership function in [30]

$$
y_{i}=\frac{1}{1+e^{\left(-x_{i}-a\right) / b}}-\frac{1}{1+e^{\left(-x_{i}-a\right) / b+a}},
$$

where $x_{i}$ and $y_{i}, i=1,2,3, \ldots, n(n \gg 3)$, are the measured data contaminated with noises and $a$ and $b$ are unknown parameters to be estimated. When the parameters $a$ and $b$ are already known, this nonlinear membership function is often used in a single-input and single-output fuzzy system.

Define the cost function and the parameter vector $\theta_{1}$ as

$$
\begin{aligned}
J_{1}(a, b) & :=\frac{1}{2} \sum_{i=1}^{n}\left(y_{i}-\frac{1}{1+e^{\left(-x_{i}-a\right) / b}}+\frac{1}{1+e^{\left(-x_{i}-a\right) / b+a}}\right)^{2}, \\
\boldsymbol{\theta}_{1} & :=[a, b]^{T} .
\end{aligned}
$$

The gradient of $J_{1}\left(\theta_{1}\right)=J_{1}(a, b)$ with respect to $\theta_{1}$ is

$$
\begin{aligned}
\nabla J_{1}\left(\boldsymbol{\theta}_{1}\right) & =\left[\begin{array}{l}
\frac{\partial J_{1}\left(\boldsymbol{\theta}_{1}\right)}{\partial a} \\
\frac{\partial J_{1}\left(\boldsymbol{\theta}_{1}\right)}{\partial b}
\end{array}\right] \\
& =\left[\begin{array}{l}
\sum_{i=1}^{n}\left(\frac{e^{-\left(x_{i}+a\right) / b}}{b\left(1+e^{\left(-x_{i}-a\right) / b}\right)^{2}}-\frac{(1-b) e^{-\left(x_{i}+a\right) / b+a}}{b\left(1+e^{\left(-x_{i}-a\right) / b}+a\right)^{2}}\right)\left(y_{i}-\frac{1}{1+e^{\left(-x_{i}-a\right) / b}}+\frac{1}{1+e^{\left(-x_{i}-a\right) / b+a}}\right) \\
\sum_{i=1}^{n}\left(\frac{\left(-x_{i}-a\right) e^{-\left(x_{i}+a\right) / b}}{b^{2}\left(1+e^{\left(-x_{i}-a\right) / b}\right)^{2}}-\frac{\left(-x_{i}-a\right) e^{-\left(x_{i}+a\right) / b+a}}{b^{2}\left(1+e^{\left(-x_{i}-a\right) / b+a}\right)^{2}}\right)\left(y_{i}-\frac{1}{1+e^{\left(-x_{i}-a\right) / b}}+\frac{1}{1+e^{\left(-x_{i}-a\right) / b+a}}\right)
\end{array}\right] .
\end{aligned}
$$

Let $k$ be the iterative variable and $\hat{\theta}_{1}^{k}=\left[\begin{array}{c}\hat{a}^{k} \\ \widehat{b}_{k}\end{array}\right]$ be the estimates of $\theta_{1}$ at iteration $k$. We can get the following gradient-based iterative algorithm:

$$
\begin{aligned}
& \widehat{\theta}_{1}^{k+1}=\widehat{\theta}_{1}^{k}-\mu_{1}^{k} \nabla J_{1}\left(\widehat{\theta}_{1}^{k}\right), \\
& \nabla J_{1}\left(\widehat{\theta}_{1}^{k}\right)=\left[\begin{array}{l}
\sum_{i=1}^{n}\left(\frac{e^{-\left(x_{i}+\widehat{a}_{k}\right) / \hat{b}_{k}}}{\widehat{b}_{k}\left(1+e^{\left(-x_{i}-\widehat{a}_{k}\right) / \hat{b}_{k}}\right)^{2}}-\frac{\left(1-\widehat{b}_{k}\right) e^{-\left(x_{i}+\widehat{a}_{k}\right) \widehat{b}_{k}+a}}{\widehat{b}_{k}\left(1+e^{\left(-x_{i}-\widehat{a}_{k}\right) \widehat{b}_{k}}+a\right)^{2}}\right) \varepsilon_{1}^{i}(k) \\
\sum_{i=1}^{n}\left(\frac{\left(-x_{i}-\widehat{a}_{k}\right) e^{-\left(x_{i}+\widehat{a}_{k}\right) \widehat{b}_{k}}}{\hat{b}_{k}^{2}\left(1+e^{\left(-x_{i}-\widehat{a}_{k}\right) \widehat{b}_{k}}\right)^{2}}-\frac{\left(-x_{i}-\widehat{a}_{k}\right) e^{-\left(x_{i}+\widehat{a}_{k}\right) \widehat{b}_{k}+a}}{\widehat{b}_{k}^{2}\left(1+e^{\left(-x_{i}-\widehat{a}_{k}\right) \widehat{b}_{k}+\widehat{a}_{k}}\right)^{2}}\right) \varepsilon_{1}^{i}(k)
\end{array}\right], \\
& \varepsilon_{1}^{i}(k)=y_{i}-\frac{1}{1+e^{\left(-x_{i}-\widehat{a}_{k}\right) \widehat{b}_{k}}}+\frac{1}{1+e^{\left(-x_{i}-\widehat{a}_{k}\right) / \widehat{b}_{k}+\widehat{a}_{k}}}, \\
& \mathbf{P}_{1}^{k}=-\nabla J_{1}\left(\widehat{\theta}_{1}^{k}\right) \text {, } \\
& \mu_{1}^{k}=\underset{\mu_{1} \geqslant 0}{\arg \min } J_{1}\left(\widehat{\theta}_{1}^{k}+\mu_{1} \mathbf{P}_{1}^{k}\right) \text {. }
\end{aligned}
$$


There exist many methods which can compute the step size $\mu_{1}^{k}$, e.g., the steepest descent method, the stochastic gradient method, and the projection method [31-35].

Define

$$
\begin{aligned}
\frac{-1}{b} & =a_{1}, \\
\frac{-a}{b} & =a_{2}, \\
\frac{-a}{b}+a & =a_{3} .
\end{aligned}
$$

Then, equation (1) can be simplified as

$$
y(t)=\frac{1}{1+e^{a_{1} x+a_{2}}}-\frac{1}{1+e^{a_{1} x+a_{3}}} .
$$

Define the cost function and the parameter vector $\theta_{2}$ as

$$
\begin{aligned}
J_{2}\left(a_{1}, a_{2}, a_{3}\right) & :=\frac{1}{2} \sum_{i=1}^{n}\left(y_{i}-\frac{1}{1+e^{\left(-x_{i}-a\right) / b}}+\frac{1}{1+e^{\left(-x_{i}-a\right) / b+a}}\right)^{2}, \\
\boldsymbol{\theta}_{2} & :=\left[a_{1}, a_{2}, a_{3}\right]^{T} .
\end{aligned}
$$

The gradient of $J_{2}\left(\theta_{2}\right)=J_{2}\left(a_{1}, a_{2}, a_{3}\right)$ with respect to $\theta_{2}$

$$
\nabla J_{2}\left(\boldsymbol{\theta}_{2}\right)=\left[\begin{array}{l}
\frac{\partial J_{2}\left(\boldsymbol{\theta}_{2}\right)}{\partial a_{1}} \\
\frac{\partial J_{2}\left(\boldsymbol{\theta}_{2}\right)}{\partial a_{2}} \\
\frac{\partial J_{2}\left(\boldsymbol{\theta}_{2}\right)}{\partial a_{3}}
\end{array}\right]=\left[\begin{array}{c}
\sum_{i=1}^{n}\left(\frac{-x_{i} e^{a_{1} x+a_{2}}}{\left(1+e^{a_{1} x_{i}+a_{2}}\right)^{2}}-\frac{-x_{i} e^{a_{1} x_{i}+a_{3}}}{\left(1+e^{a_{1} x_{i}+a_{3}}\right)^{2}}\right)\left(y_{i}-\frac{1}{1+e^{a_{1} x_{i}+a_{2}}}-\frac{1}{1+e^{a_{1} x_{i}+a_{3}}}\right) \\
\sum_{i=1}^{n} \frac{-e^{a_{1} x_{i}+a_{2}}}{\left(1+e^{a_{1} x_{i}+a_{2}}\right)^{2}}\left(y_{i}-\frac{1}{1+e^{a_{1} x_{i}+a_{2}}}-\frac{1}{1+e^{a_{1} x_{i}+a_{3}}}\right) \\
\sum_{i=1}^{n} \frac{e^{a_{1} x_{i}+a_{3}}}{\left(1+e^{a_{1} x_{i}+a_{3}}\right)^{2}}\left(y_{i}-\frac{1}{1+e^{a_{1} x_{i}+a_{2}}}-\frac{1}{1+e^{a_{1} x_{i}+a_{3}}}\right)
\end{array}\right] .
$$

Let $k$ be the iterative variable and $\hat{\theta}_{2}^{k}=\left[\begin{array}{c}\hat{a}_{1}^{k} \\ \hat{a}_{2}^{k} \\ \hat{a}_{3}^{k}\end{array}\right]$ be the estimates of $\theta_{2}$ at iteration $k$. We can get the following gradient-based iterative algorithm:

$$
\begin{aligned}
& \widehat{\theta}_{2}^{k+1}=\widehat{\theta}_{2}^{k}-\mu_{2}^{k} \nabla J_{2}\left(\widehat{\theta}_{2}^{k}\right), \\
& \varepsilon_{2}^{i}(k)=y_{i}-\frac{1}{1+e^{a_{1}{ }^{k} x_{i}+\widehat{a}_{2}{ }^{k}}}-\frac{1}{1+e^{\widehat{a}_{1}^{k} x_{i}+a_{3}}}, \\
& {\left[\sum_{i=1}^{n}\left(\frac{-x_{i} e^{\hat{a}_{1}^{k} x_{i}+\vec{a}_{2}^{k}}}{\left(1+e^{\hat{a_{1}} x_{i}+a_{2}^{k}}\right)^{2}}-\frac{-x_{i} e^{\hat{a}_{1}^{k} x_{i}+\hat{a}_{3}^{k}}}{\left(1+e^{\hat{a}_{1}^{k} x_{i}+\vec{a}_{3}^{k}}\right)^{2}}\right) \varepsilon_{i}(k)\right.} \\
& \nabla J_{2}\left(\hat{\theta}_{2}^{k}\right)=\quad \sum_{i=1}^{n} \frac{-e^{-\hat{a}_{1}^{k} x_{i}+\hat{a}_{2}^{k}}}{\left(1+e^{\hat{a}_{1}^{k} x_{i}+\hat{a}_{2}^{k}}\right)^{2}} \varepsilon_{i}(k) \\
& \sum_{i=1}^{n} \frac{e^{\vec{a}_{1}^{k} x_{i}+a_{3}^{k}}}{\left(1+e^{\vec{a}_{1}^{k} x_{i}+a_{3}^{k}}\right)^{2}} \varepsilon_{i}(k) \\
& \mathbf{P}_{2}^{k}=-\nabla J_{2}\left(\widehat{\theta}_{2}^{k}\right) \text {, } \\
& \mu_{2}^{k}=\underset{\mu_{2} \geqslant 0}{\arg \min } J_{2}\left(\hat{\theta}_{2}^{k}+\mu_{2} \mathbf{P}_{2}^{k}\right) \text {. }
\end{aligned}
$$

The above iterative algorithms are difficult and have heavy computational burden because they update the parameters with all the collected data. In order to reduce the computational effort, we propose two modified methods in the next section.

\section{The Model Transformation-Based Stochastic Gradient Method and Iterative Method}

Convert the nonlinear function (6) to an identification model:

$$
y=-\left(e^{a_{2}}+e^{a_{3}}\right) y e^{a_{1} x}+\left(e^{a_{3}}-e^{a_{2}}\right) e^{a_{1} x}-e^{a_{2}+a_{3}} y e^{2 a_{1} x} .
$$

Then define the parameter vector $\theta_{3}$ and the information vector $\varphi_{3}(t)$ as

$$
\begin{gathered}
\boldsymbol{\theta}_{3}:=\left[\begin{array}{c}
e^{a_{2}}+e^{a_{3}} \\
e^{a_{3}}-e^{a_{2}} \\
e^{a_{2}+a_{3}}
\end{array}\right], \\
\boldsymbol{\varphi}_{3}(t):=\left[\begin{array}{c}
-y(t) e^{a_{1} x(t)} \\
e^{a_{1} x(t)} \\
-y(t) e^{2 a_{1} x(t)}
\end{array}\right] .
\end{gathered}
$$

Without loss of generality, a noise term $v(t)$ with zero mean is introduced to the identification model in (10), and the identification model is put into a concise form

$$
y(t)=\boldsymbol{\varphi}_{3}^{T}(t) \boldsymbol{\theta}_{3}+v(t) .
$$


Let $\widehat{\theta}_{3}(i)$ be the $i$ th element of the vector $\widehat{\theta}_{3}$; we have $\widehat{a}_{2}=\ln \left(\widehat{\theta}_{3}(1)-\widehat{\theta}_{3}(2) / 2\right), \quad \widehat{a}_{3}=\ln \left(\widehat{\theta}_{3}(1)+\widehat{\theta}_{3}(2) / 2\right), \quad$ and $\widehat{a}=\widehat{a}_{3}-\widehat{a}_{2}$. At last, with the estimated parameters $\widehat{a}$ and $\widehat{a}_{2}$, we obtain $\widehat{b}=-\left(\widehat{a} / \widehat{a}_{2}\right)$.

Let $\widehat{\theta}_{3}(t)$ and $\widehat{\varphi}_{3}(t)$ be the estimate of $\theta_{3}$ and $\varphi_{3}$, and $\widehat{\theta}_{3}(t)$ and $\widehat{\varphi}_{3}(t)$ are defined as

$$
\begin{aligned}
& \widehat{\theta}_{3}(t)=\left[\begin{array}{c}
e^{\widehat{a}_{2}(t)}+e^{\widehat{a}_{3}(t)} \\
e^{\widehat{a}_{3}(t)}-e^{\widehat{a}_{2}(t)} \\
e^{\widehat{a}_{2}(t)+\widehat{a}_{3}(t)}
\end{array}\right], \\
& \widehat{\varphi}_{3}(t)=\left[\begin{array}{c}
y(t) e^{\widehat{a}_{1}(t-1) x(t)} \\
e^{\widehat{a}_{1}(t-1) x(t)} \\
y(t) e^{2 \widehat{a}_{1}(t-1) x(t)}
\end{array}\right] .
\end{aligned}
$$

Using the gradient search and minimizing the quadratic criterion function

$$
J_{3}\left(\boldsymbol{\theta}_{3}\right):=\left\|y(t)-\boldsymbol{\varphi}_{3}^{T}(t) \boldsymbol{\theta}_{3}\right\|^{2}
$$

lead to the model transformation-based stochastic gradient (MT-SG) algorithm:

$$
\begin{gathered}
\widehat{\theta}_{3}(t)=\widehat{\theta}_{3}(t-1)+\widehat{\varphi}_{3}(t) \mu_{3}(t)\left[y(t)-\widehat{\varphi}_{3}^{T}(t) \widehat{\theta}_{3}(t-1)\right] \\
\widehat{a}_{1}(t-1)=\frac{\widehat{a}_{2}(t-1)}{\hat{a}_{3}(t-1)-\widehat{a}_{2}(t-1)} \\
\widehat{\varphi}_{3}(t)=\left[\begin{array}{c}
y(t) e^{\widehat{a}_{1}(t-1) x(t)} \\
e^{\widehat{a}_{1}(t-1) x(t)} \\
y(t) e^{2 \widehat{a}_{1}(t-1) x(t)}
\end{array}\right] \\
0<\mu_{3}(t)<\frac{2}{\lambda_{\max }\left[\widehat{\varphi}_{3}^{T}(t) \widehat{\varphi}_{3}(t)\right]}
\end{gathered}
$$

The steps of computing the parameter estimation vector $\widehat{\theta}_{3}(t)$ by the MT-SG algorithm in (15)-(18) are listed as follows:

(1) Collect the measured data $\{x(t), y(t): t=0,1,2, \ldots\}$

(2) To initialize, let $t=1$ and $\widehat{\theta}_{3}(0)=\mathbf{1} / p_{0}$

(3) Compute $\widehat{a}_{1}(t-1)$ according to (16)

(4) Compute $\widehat{\varphi}_{3}(t)$ by (17)

(5) Choose $\mu_{3}(t)$ according to (18)

(6) Update the parameter estimation vector $\widehat{\theta}_{3}(t)$ by (15), and compare $\widehat{\theta}_{3}(t)$ and $\widehat{\theta}_{3}(t-1)$ : if they are sufficiently close, or for some preset small $\varepsilon$, if $\left\|\widehat{\theta}_{3}(t)-\widehat{\theta}_{3}(t-1)\right\| \leqslant \varepsilon$, then terminate the procedure and obtain the estimate $\widehat{\theta}_{3}(t)$; otherwise, increase $t$ by 1 and go to step 3

In general, the MT-SG algorithm is suitable for online identification and the iterative algorithm is used for offline identification. The iterative algorithm employs the idea of updating the estimate $\hat{\theta}_{3}$ using a fixed data batch with a finite length $L$ and thus has a higher estimation accuracy than the SG algorithm. Next, we use finite measurement input-output data $\left\{x_{i}, y_{i}, i=0,1, \ldots, L\right\}$ and iterative with subscript $k$.

Define the stacked output vector $\mathbf{Y}(L)$ and the stacked information matrix $\Phi(L)$ as

$$
\begin{aligned}
\mathbf{Y}(L) & :=[y(L), y(L-1), y(L-2), \ldots, y(1)]^{T} \in \mathbb{R}^{L}, \\
\boldsymbol{\Phi}(L) & :=[\boldsymbol{\varphi}(L), \boldsymbol{\varphi}(L-1), \boldsymbol{\varphi}(L-2), \ldots, \boldsymbol{\varphi}(1)]^{T} \in \mathbb{R}^{L \times 3} .
\end{aligned}
$$

Let $\hat{\theta}_{3}^{k}$ and $\hat{\varphi}_{3}^{k}$ be the iterative estimate of $\theta_{3}$ and $\varphi_{3}$ at iteration $k=1,2,3, \ldots$, and $\widehat{\theta}_{3}^{k}, \widehat{\varphi}_{3}^{k}$, and $\Phi_{3}^{k}(L)$ are defined as

$$
\begin{aligned}
\hat{\theta}_{3}^{k} & =\left[e^{\hat{a}_{3}^{k}}+e^{\vec{a}_{3}^{k}}, e^{\widehat{a}_{3}^{k}}-e^{\vec{a}_{2}^{k}}, e^{\hat{a}_{2}^{k}+a_{3}^{k}}\right]^{T}, \\
\widehat{\varphi}_{3}^{k}(L) & =\left[y(L) e^{\widehat{a}_{1}^{k-1} x(L)}, e^{\hat{a}_{1}^{k-1} x(L)}, y(L) e^{2 a_{1}^{k-1} x(L)}\right]^{T}, \\
\Phi_{3}^{k}(L) & =\left[\varphi_{3}^{k}(L), \varphi_{3}^{k}(L-1), \varphi_{3}^{k}(L-2), \ldots, \varphi_{3}^{k}(1)\right]^{T} \in \mathbb{R}^{L \times 3} .
\end{aligned}
$$

Define a quadratic criterion function

$$
J_{4}\left(\boldsymbol{\theta}_{3}\right):=\left\|\mathbf{Y}(L)-\boldsymbol{\Phi}_{3}(L) \boldsymbol{\theta}_{3}\right\|^{2} .
$$

Minimizing $J_{4}\left(\theta_{3}\right)$ and using the negative gradient search lead to the model transformation-based gradient iterative algorithm (MT-GI) of computing $\theta_{3}$ :

$$
\begin{aligned}
\hat{\theta}_{3}^{k} & =\hat{\theta}_{3}^{k-1}+\mu_{3}^{k} \Phi_{3}^{k T}(L)\left[\mathbf{Y}(L)-\Phi_{3}^{k}(L) \hat{\theta}_{3}^{k-1}\right] \\
\hat{a}_{1}^{k-1} & =\frac{\widehat{a}_{2}^{k-1}}{\hat{a}_{3}^{k-1}-\hat{a}_{2}^{k-1},} \\
\hat{\varphi}_{3}^{k}(L) & =\left[y(L) e^{\widehat{a}_{1}^{k-1} x(L)}, e^{\hat{a}_{1}^{k-1} x(L)}, y(L) e^{2 \hat{a}_{1}^{k-1} x(L)}\right]^{T} \\
\boldsymbol{\Phi}_{3}^{k}(L) & =\left[\varphi_{3}^{k}(L), \varphi_{3}^{k}(L-1), \varphi_{3}^{k}(L-2), \ldots, \varphi_{3}^{k}(1)\right]^{T} \in \mathbb{R}^{L \times 3}, \\
0<\mu_{3}^{k} & <\frac{2}{\lambda_{\max }\left[\widehat{\Phi}_{3}^{k T}(L) \widehat{\Phi}_{3}^{k}(L)\right]} .
\end{aligned}
$$

The steps of computing the parameter estimation matrix $\widehat{\theta}_{3}^{k}$ by the MT-GI algorithm are listed as follows:

(1) Collect the input data $\{x(t), y(t): t=0,1,2, \ldots, L\}$

(2) To initialize, let $k=1$ and $\widehat{\theta}_{3}^{0}=\mathbf{1} / p_{0}$, with $\mathbf{1}$ being a column vector whose entries are all unity and $p_{0}=10^{6}$.

(3) Compute $\hat{a}_{1}^{k-1}$ by (23)

(4) Form $\widehat{\varphi}_{3}^{k}(t)$ according to (24) and $\widehat{\Phi}_{3}^{k}(L)$ according to $(25)$

(5) Choose a maximum $\mu_{3}^{k}$ by (26)

(6) Compute $\widehat{\theta}_{3}^{k}$ by (22) 
TABle 1: The MT-SG estimates and errors.

\begin{tabular}{|c|c|c|c|c|c|}
\hline$t$ & $c_{1}$ & $c_{2}$ & $c_{3}$ & $c_{4}$ & $\delta(\%)$ \\
\hline 10 & 0.71448 & 0.48237 & 0.95045 & -0.83293 & 49.87382 \\
\hline 20 & 1.13511 & 0.48237 & 1.07447 & -0.83293 & 33.91638 \\
\hline 30 & 1.20307 & 0.48237 & 0.83073 & -0.83293 & 25.69778 \\
\hline 50 & 1.44768 & 0.48237 & 0.77121 & -0.83293 & 13.64527 \\
\hline 100 & 1.67539 & 0.48237 & 0.68940 & -0.83293 & 2.15313 \\
\hline 150 & 1.70569 & 0.48237 & 0.67428 & -0.83293 & 0.89147 \\
\hline 200 & 1.71124 & 0.48237 & 0.67039 & -0.83293 & 0.81363 \\
\hline 250 & 1.71176 & 0.48237 & 0.67022 & -0.83293 & 0.81274 \\
\hline 300 & 1.71176 & 0.48237 & 0.67022 & -0.83293 & 0.81274 \\
\hline True values & 1.71200 & 0.49900 & 0.67000 & -0.83000 & \\
\hline
\end{tabular}

TABLE 2: The MT-GI estimates and errors with $L=20$.

\begin{tabular}{|c|c|c|c|c|c|}
\hline$k$ & $c_{1}$ & $c_{2}$ & $c_{3}$ & $c_{4}$ & $\delta(\%)$ \\
\hline 10 & 1.54756 & 0.05391 & 0.49679 & -0.83293 & 24.30909 \\
\hline 20 & 1.69311 & 0.08402 & 0.65245 & -0.83293 & 20.00987 \\
\hline 30 & 1.71353 & 0.10939 & 0.67933 & -0.83293 & 18.75623 \\
\hline 50 & 1.71681 & 0.15797 & 0.68337 & -0.83293 & 16.42697 \\
\hline 100 & 1.71564 & 0.27616 & 0.68055 & -0.83293 & 10.73834 \\
\hline 150 & 1.71441 & 0.39041 & 0.67768 & -0.83293 & 5.24201 \\
\hline 200 & 1.71322 & 0.50085 & 0.67491 & -0.83293 & 0.29525 \\
\hline True values & 1.71200 & 0.49900 & 0.67000 & -0.83000 & \\
\hline
\end{tabular}

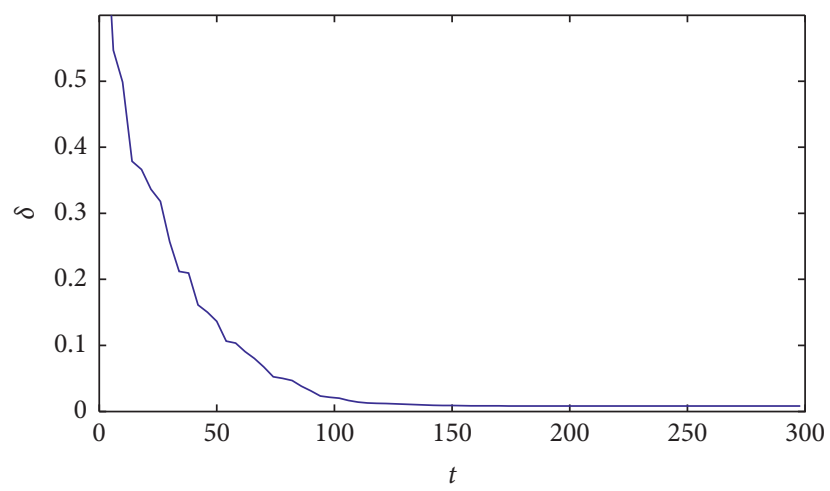

FIgURE 1: The parameter estimation errors $\delta$ versus $t$ (MT-SG).

(7) Compare $\widehat{\theta}_{3}^{k}$ and $\widehat{\theta}_{3}^{k-1}$ : if they are sufficiently close, or for some preset small $\varepsilon$, if $\left\|\widehat{\theta}_{3}^{k}-\widehat{\theta}_{3}^{k-1}\right\| \leqslant \varepsilon$, then terminate the procedure and obtain the iterative times $k$, and estimate $\widehat{\theta}_{3}^{k}$; otherwise, increase $k$ by 1 and go to step 3

\section{Example}

Consider a nonlinear function

$$
y=\frac{1}{1+e^{-(x+a) / b}}-\frac{1}{1+e^{-(x+a) / b+a}}+v=\frac{1}{1+e^{-(x+0.6) / 1.2}}-\frac{1}{1+e^{-(x+0.6) / 1.2+0.6}}+v
$$

where $\quad a_{1}=(-1 / b)=0.83, a_{2}=(-a / b)=-0.5$, and $a_{3}=(-a / b)+a=0.1$, and then, we can conclude

$$
\boldsymbol{\theta}_{3}=\left[\begin{array}{c}
e^{a_{2}}+e^{a_{3}} \\
e^{a_{3}}-e^{a_{2}} \\
e^{a_{2}+a_{3}}
\end{array}\right]=\left[\begin{array}{c}
1.712 \\
0.499 \\
0.67
\end{array}\right]
$$




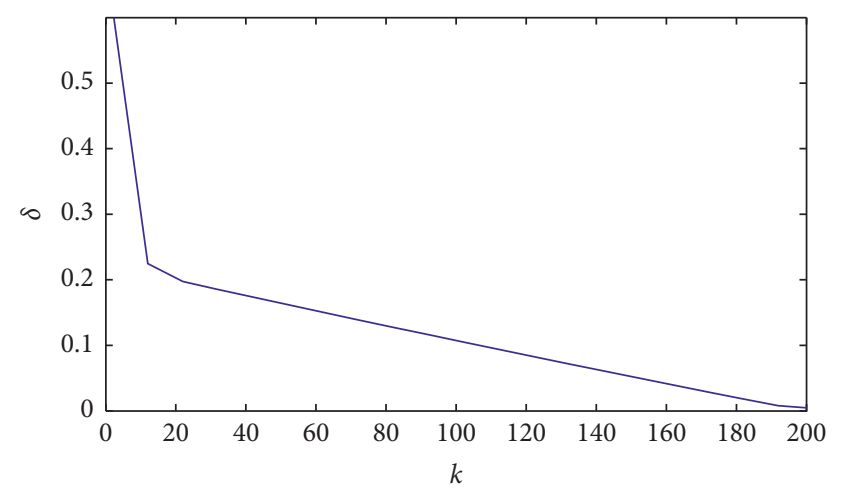

Figure 2: The parameter estimation errors $\delta$ versus $k$ (MT-GI).

Define

$$
\left[\begin{array}{c}
c_{1} \\
c_{2} \\
c_{3} \\
c_{4}
\end{array}\right]:=\left[\begin{array}{c}
e^{a_{2}}+e^{a_{3}} \\
e^{a_{3}}-e^{a_{2}} \\
e^{a_{2}+a_{3}} \\
a_{1}
\end{array}\right]=\left[\begin{array}{c}
1.712 \\
0.499 \\
0.67 \\
-0.83
\end{array}\right]
$$

Assume $x(t)$ is the input and is taken as a persistent excitation signal with zero mean and unit variances and $v(t)$ is taken as white noise with a zero mean and variance $\sigma_{1}^{2}=0.10^{2}$. Applying the MT-SG algorithm and MT-GI algorithm to estimate the parameters of this system, the parameter estimates and their errors are shown in Tables 1 and 2, and the parameter estimation errors $\delta:=\left\|\widehat{\theta}_{3}-\theta_{3}\right\| /\left\|\theta_{3}\right\|$ versus $t$ and $k$ are shown in Figures 1 and 2 .

From Tables 1 and 2 and Figures 1 and 2, we can draw the following conclusions:

(1) The MT-GI algorithm has higher estimation accuracy than the MT-SG algorithm

(2) The parameter estimation errors by the MT-SG algorithm become smaller and smaller and go to zero with $t$ increasing

(3) The parameter estimation errors by the MT-GI algorithm become smaller and smaller and go to zero with the subscript $k$ increasing

\section{Conclusions}

This paper presents two identification methods for a nonlinear membership function. An equation converted method is proposed to convert the nonlinear function to a concise model, and then an MT-SG algorithm and an MTGI algorithm are provided to identify the nonlinear function. The simulation results verify the proposed algorithms.

\section{Data Availability}

The simulation data used to support the findings of this study are available from the corresponding author upon request.

\section{Conflicts of Interest}

The authors declare that they have no conflicts of interest.

\section{Acknowledgments}

This work was supported by the Natural Science Foundation of Jiangsu Province (no. BK20131109).

\section{References}

[1] H. Li, J. Bai, S. Kanae, Y. Li, and L. Yue, “Active disturbance rejection control of three-phase grid-connected photovoltaic systems," International Journal of Modelling, Identification and Control, vol. 33, no. 3, pp. 225-236, 2019.

[2] P. Li and W. Zhang, "U-model enhanced MIMO decoupling control of thickness and plate type of cold rolling temper mill," International Journal of Modelling, Identification and Control, vol. 33, no. 3, pp. 189-197, 2019.

[3] H. Xu, F. Ding, and E. Yang, "Three-stage multi-innovation parameter estimation for an exponential autoregressive timeseries model with moving average noise by using the data filtering technique," International Journal of Robust and Nonlinear Control, vol. 31, no. 1, pp. 166-184, 2021.

[4] L. Xu, F. Ding, and E. Yang, "Auxiliary model multiinnovation stochastic gradient parameter estimation methods for nonlinear sandwich systems," International Journal of Robust and Nonlinear Control, vol. 31, no. 1, pp. 148-165, 2021.

[5] J. Chen, Q. Zhu, and Y. Liu, "Modified Kalman filtering based multi-step-length gradient iterative algorithm for ARX models with random missing outputs," Automatica, vol. 118, Article ID 109034, 2020.

[6] G. Y. Chen and M. Gan, "A regularized variable projection algorithm for separable nonlinear least squares problems," IEEE Transactions on Automatic Control, vol. 64, no. 2, pp. 526-537, 2019.

[7] Q. M. Zhu, "An implicit least squares algorithm for nonlinear rational model parameter estimation," Applied Mathematical Modelling, vol. 29, no. 7, pp. 673-689, 2005.

[8] D. Wang, L. Li, Y. Ji, and Y. Yan, "Model recovery for Hammerstein systems using the auxiliary model based orthogonal matching pursuit method," Applied Mathematical Modelling, vol. 54, pp. 537-550, 2018.

[9] F. Ding, L. Lv, J. Pan, X. Wan, and X.-B. Jin, "Two-stage gradient-based iterative estimation methods for controlled autoregressive systems using the measurement data," International Journal of Control, Automation and Systems, vol. 18, no. 4, pp. 886-896, 2020.

[10] D. Wang, L. Mao, and F. Ding, "Recasted models-based hierarchical extended stochastic gradient method for MIMO nonlinear systems," IET Control Theory \& Applications, vol. 11, no. 4, pp. 476-485, 2017.

[11] Y. Gu, Q. M. Zhu, J. C. Liu et al., "Multi-innovation stochastic gradient parameter and state estimation algorithm for dualrate state-space systems with d-step time delay," Complexity, vol. 2020, Article ID 6128697, 11 pages, 2020.

[12] J. H. Li and X. Li, "Particle swarm optimization iterative identification algorithm and gradient iterative identification algorithm for wiener systems with colored noise," Complexity, vol. 2018, Article ID 7353171, 8 pages, 2018.

[13] Q. Tu, Y. J. Rong, and J. Chen, "Parameter identification of ARX models based on modified momentum gradient descent 
algorithm," Complexity, vol. 2020, Article ID 9537075 , 11 pages, 2020.

[14] D. Wang, S. Zhang, M. Gan, and J. Qiu, "A novel EM identification method for Hammerstein systems with missing output data," IEEE Transactions on Industrial Informatics, vol. 16, no. 4, pp. 2500-2508, 2020.

[15] J. Chen, X. Wang, and R. Ding, "Gradient based estimation algorithm for Hammerstein systems with saturation and dead-zone nonlinearities," Applied Mathematical Modelling, vol. 36, no. 1, pp. 238-243, 2012.

[16] J. Li, R. Ding, and Y. Yang, "Iterative parameter identification methods for nonlinear functions," Applied Mathematical Modelling, vol. 36, no. 6, pp. 2739-2750, 2012.

[17] R. J. Wai, M. A. Kuo, and J. D. Lee, "Design of cascade adaptive fuzzy sliding-mode control for nonlinear two-axis inverted-pendulum servomechanism," IEEE Transactions on Fuzzy Systems, vol. 16, no. 5, pp. 1232-1244, 2008.

[18] Y. C. Hsueh, S. F. Su, C. W. Tao, and C. C. Hsiao, "Robust $L_{2}$-gain compensative control for direct adaptive fuzzy-control-system design," IEEE Transactions on Fuzzy Systems, vol. 18, no. 4, pp. 661-673, 2010.

[19] S. C. Tong, Y. Li, Y. M. Li, and Y. J. Liu, "Observer-based adaptive fuzzy backstepping control for a class of stochastic nonlinear strict-feedback systems," IEEE Transactions on Systems, Man, and Cybernetics, vol. 41, no. 6, pp. 1693-1704, 2011.

[20] L.-X. Wang and J. M. Mendel, "Fuzzy basis functions, universal approximation, and orthogonal least-squares learning," IEEE Transactions on Neural Networks, vol. 3, no. 5, pp. 807-814, 1992.

[21] K.-H. Cheng, C.-F. Hsu, C.-M. Lin, T.-T. Lee, and C. Li, "Fuzzy-neural sliding-mode control for DC-DC converters using asymmetric Gaussian membership functions," IEEE Transactions on Industrial Electronics, vol. 54, no. 3, pp. 1528-1536, 2007.

[22] D. Gu and H. Hu, "Using fuzzy logic to design separation function in flocking algorithms," IEEE Transactions on Fuzzy Systems, vol. 16, no. 4, pp. 826-838, 2008.

[23] X. J. Zeng and M. G. Singh, "Approximation theory of fuzzy systems-SISO case," IEEE Transactions on Fuzzy Systems, vol. 2, no. 2, pp. 162-176, 1994.

[24] Y. Yam, M. L. Wong, and P. Baranyi, "Interpolation with function space representation of membership functions," IEEE Transactions on Fuzzy Systems, vol. 14, no. 3, pp. 398411, 2006.

[25] J. L. Ding, "The hierarchical iterative identification algorithm for multi-input-output-error systems with autoregressive noise," Complexity, vol. 2017, Article ID 5292894, 11 pages, 2017.

[26] D. Wang, Y. Yan, Y. Liu, and J. Ding, "Model recovery for Hammerstein systems using the hierarchical orthogonal matching pursuit method," Journal of Computational and Applied Mathematics, vol. 345, pp. 135-145, 2019.

[27] G.-Y. Chen, M. Gan, C. L. P. Chen, and H.-X. Li, "Basis function matrix-based flexible coefficient autoregressive models: a framework for time series and nonlinear system modeling," IEEE Transactions on Cybernetics, vol. 51, no. 2, p. 614, 2021.

[28] J. Pan, H. Ma, X. Jiang et al., "Adaptive gradient-based iterative algorithm for multivariabe controlled autoregressive moving average systems using the data filtering technique," Complexity, vol. 2018, Article ID 9598307, 11 pages, 2018.
[29] D. Wang, Z. Zhang, and B. Xue, "Decoupled parameter estimation methods for Hammerstein systems by using filtering technique," IEEE Access, vol. 6, pp. 66612-66620, 2018.

[30] Z. H. Mao, Y. D. Li, and X. F. Zhang, "Approximation capability of fuzzy systems using translations and dilations of one fixed function as membership functions," IEEE Transactions on Fuzzy Systems, vol. 5, no. 3, pp. 468-473, 1997.

[31] M. Gan, Y. Guan, G.-Y. Chen, and C. L. P. Chen, "Recursive variable projection algorithm for a class of separable nonlinear models," IEEE Transactions on Neural Networks and Learning Systems, pp. 1-12, 2020.

[32] G.-Y. Chen, M. Gan, F. Ding, and C. L. P. Chen, "Modified gram-schmidt method-based variable projection algorithm for separable nonlinear models," IEEE Transactions on Neural Networks and Learning Systems, vol. 30, no. 8, pp. 2410-2418, 2019.

[33] M. Gan, C. L. P. Chen, G.-Y. Chen, and L. Chen, "On some separated algorithms for separable nonlinear least squares problems," IEEE Transactions on Cybernetics, vol. 48, no. 10, pp. 2866-2874, 2018.

[34] S. Fan, L. Xu, F. Ding, A. Alsaedi, and T. Hayat, "Correlation analysis-based stochastic gradient and least squares identification methods for errors-in-variables systems using the multiinnovation," International Journal of Control, Automation and Systems, vol. 19, no. 1, pp. 289-300, 2021.

[35] F. Ding, L. Xu, D. Meng, X.-B. Jin, A. Alsaedi, and T. Hayat, "Gradient estimation algorithms for the parameter identification of bilinear systems using the auxiliary model," Journal of Computational and Applied Mathematics, vol. 369, Article ID 112575, 2020. 